\title{
A Phase I Study of the Pharmacokinetics and Pharmacodynamics of Intranasal Doxylamine in Subjects with Chronic Intermittent Sleep Impairment
}

\author{
Mark Allison ${ }^{1}$ Cecilia Hale ${ }^{2}$
}

Published online: 18 April 2018

(C) The Author(s) 2018

\begin{abstract}
Introduction Doxylamine tablets are approved as an overthe-counter sleep aid. We developed a doxylamine succinate intranasal metered-dose delivery system with the expectation of a more rapid onset of action with reduced side-effect potential compared with the oral tablet.

Methods This phase I study randomized 24 adults with chronic intermittent sleep impairment to receive either single doses of intranasal doxylamine succinate 3.2, 6.3, or $12.7 \mathrm{mg}$ or doxylamine succinate $25 \mathrm{-mg}$ oral tablet. Doxylamine pharmacokinetics were assessed using noncompartmental methods; pharmacodynamics were evaluated using the Karolinska Sleepiness Scale (KSS) and numerous psychomotor tests. Adverse events (AEs) were monitored.

Results None of the intranasal dose levels produced a mean maximum plasma concentration $\left(C_{\max }\right)$ above the $50 \mathrm{ng} / \mathrm{mL}$ target level or a time to maximum concentration shorter than that of the oral tablet. At the highest intranasal dose, $C_{\max }$ and area under the doxylamine concentrationtime curve were approximately $25 \%$ of the values achieved with the oral dose. Variation in most pharmacokinetic parameters was higher with intranasal compared with oral dosing. A relationship between plasma doxylamine concentration and KSS change from baseline was evident for the 25-mg tablet and, to a lesser extent, for the 12.7-mg intranasal dose. Changes from baseline in psychomotor parameters did not show a relationship to intranasal dose,
\end{abstract}

and did not distinguish between intranasal versus oral dosing. The most common AEs with intranasal dosing were nasal congestion, nasal dryness, and frontal headache.

Conclusion The nasal spray did not increase doxylamine absorption or systemic bioavailability compared with the oral tablet.

\section{Key Points}

An intranasal formulation of the sleep aid doxylamine succinate was developed for the purpose of delivering a more rapid onset of sleep with minimal psychomotor impairment upon awakening than that provided by the currently approved $25-\mathrm{mg}$ oral tablet.

However, at the doses tested, intranasal doxylamine did not increase the absorption or systemic bioavailability of doxylamine succinate compared with the 25-mg oral tablet.

The intranasal spray was well tolerated; the most common adverse events were nasal congestion, nasal dryness, and frontal headache, which were all mild in intensity and transient or intermittent.

Mark Allison

mark.allison@celerion.com

1 Clinical Research Department, Celerion, 2420 W. Baseline

Rd., Tempe, AZ 85283, USA

2 Biometrics, Hurley Consulting Associates, Summit, NJ, USA 


\section{Introduction}

Doxylamine succinate is a first-generation $\mathrm{H}_{1}$-antihistamine with nonspecific anticholinergic and sedative effects that was approved by the US Food and Drug Administration in 1978 as an over-the-counter (OTC) sleep aid [1-3]. The approved OTC product $\left(\right.$ Unisom $^{\circledR}$ SleepTabs; Chattem, Inc., Chattanooga, TN, USA) is a tablet for oral administration, recommended for use $30 \mathrm{~min}$ before bedtime. Nevertheless, clinical studies supporting the efficacy and safety of orally administered doxylamine succinate are limited [3, 4]. In a randomized, double-blind, crossover study, doxylamine at doses of 25 and $50 \mathrm{mg}$ was superior to placebo, with no difference between dose levels, in promoting sleep induction and increasing sleep duration in subjects accustomed to taking a nighttime sleep medication [1].

We sought to develop a new formulation of doxylamine that would deliver the benefits of rapid onset of sleep (i.e., reduced sleep latency) with minimal psychomotor impairment upon awakening. Pelser et al., in their preclinical study conducted in Sprague-Dawley rats, reported that the time to maximum plasma concentrations of doxylamine succinate occurred three times faster and bioavailability was almost three times higher following intranasal administration compared with oral dosing [5]. These findings suggested that doxylamine succinate is rapidly and effectively absorbed from the nasal mucosa, and prompted development of a new metered-dosing delivery system for nasal administration. The intranasal delivery system was designed with the expectation that absorption following nasal dosing would occur more rapidly and with less firstpass metabolism compared with the existing OTC tablets and, consequently, would allow effective drug concentrations in the brain to be achieved more rapidly, leading to a faster onset of action. Due to the faster absorption, it was anticipated that lower doses of nasally administered doxylamine succinate could be used, offering the potential for reduced side effects.

Herein, we report results from the initial dose-ranging pharmacokinetic study that was designed to identify the most appropriate dose of the nasal formulation to use for evaluating its efficacy and tolerability in sleep induction. The primary objective of the study was to obtain pharmacokinetic profiles and estimate pharmacokinetic parameters for three different nasal doses of the doxylamine succinate metered-dosing delivery system compared with those for the existing 25-mg oral tablet. A secondary objective was to compare the nasal and oral doses on psychomotor parameters. Safety was also evaluated.

\section{Methods}

Healthy male and female subjects aged 18-60 years were eligible if they had a self-reported history of chronic intermittent difficulty with sleep at night for 2-5 nights per week for at least 1 week per month over the last 3 months, and agreed to not use caffeine-containing products starting $24 \mathrm{~h}$ before the first dose of study medication and continuing throughout the entire study period. Eligible females of childbearing potential were required to practice an effective method of contraception. Subjects with a history of glaucoma, respiratory disease, or diabetes mellitus were excluded, as were men with difficulty urinating due to an enlarged prostate and women who were pregnant or breastfeeding. Other exclusionary criteria were use of nicotine-containing products, hemoglobin level $<12.0 \mathrm{~g} /$ $\mathrm{dL}$, blood donation within the past 2 months, and alcohol or substance abuse within the past 2 years. Prescription medications were not allowed within the past 30 days except for stable doses of lipid-lowering drugs, antihypertensive drugs, and oral contraceptives. Herbal or nutritional supplements except for vitamins and minerals were prohibited within 7 days, and any OTC medication was prohibited within $48 \mathrm{~h}$ before the first study session.

\subsection{Study Design}

This phase I, open-label, 4-arm crossover study (GSK study number V7341104) was conducted in December 2010 at Celerion, Inc. (Tempe, AZ, USA). The study consisted of four dosing periods, each separated by a washout of $72 \mathrm{~h}$. Eligible subjects entered the study site on the day before the first dosing period and remained confined to the site for the entire study. Subjects who took any antihistamine or sleep medication required a washout period of at least 10 days before admission to the study site. Adult subjects were randomly assigned (1:1:1:1) to treatment sequence and received one study treatment in each dosing period, which included doxylamine succinate 3.2, 6.3 , or $12.7 \mathrm{mg}$ delivered by intranasal metered-dose spray or a doxylamine succinate $25-\mathrm{mg}$ oral tablet. The intranasal delivery product contained doxylamine succinate at a concentration of $31.7 \mathrm{mg} / \mathrm{mL}$. Each metered dose of spray $(0.1 \mathrm{~mL})$ delivered $3.2 \mathrm{mg}$ of doxylamine succinate to the nasal cavity. Study medication was administered as one spray in one nostril for the 3.2-mg dose, one spray in each nostril for the $6.3-\mathrm{mg}$ dose, and two sprays in each nostril for the $12.7-\mathrm{mg}$ dose. Study treatments were administered in the morning after an overnight fast. The randomization schedule was provided by the Biostatistics Department of GlaxoSmithKline Consumer Healthcare. 
The study was conducted in accordance with ethical principles originating in the Declaration of Helsinki and in compliance with the International Council for Harmonisation Guideline for Good Clinical Practice and all applicable regulations. The study protocol was approved by Celerion Institutional Review Board before subjects were enrolled. All subjects provided written informed consent before participating.

\subsection{Pharmacokinetics}

Blood samples ( $3 \mathrm{~mL}$ each) were collected before and at $10,15,20,30,45,60,75,90,120$, and $150 \mathrm{~min}$, and 3, 4, 5, $6,8,12$, and $24 \mathrm{~h}$ after administration of study medication in vacutainers containing potassium ethylenediaminetetraacetic acid as anticoagulant. The tubes were mixed by inversion, placed in an ice-water bath, and centrifuged at $1300 \times g$ for $10 \mathrm{~min}$ at $5^{\circ} \mathrm{C}$. The plasma was divided into two equal aliquots and stored frozen at $-20{ }^{\circ} \mathrm{C}$ in highdensity propylene tubes. Plasma doxylamine concentrations were determined using a validated liquid chromatography-tandem mass spectrometry method having a lower limit of quantification of $0.2 \mathrm{ng} / \mathrm{mL}$. Pharmacokinetic parameters were calculated using noncompartmental methods on Phoenix ${ }^{\circledR}$ WinNonlin ${ }^{\circledR}$ version 5.1 software (Certara USA Inc., Princeton, NJ, USA). Variables included the area under the plasma doxylamine concentrationtime curve from time zero to $8 \mathrm{~h}\left(\mathrm{AUC}_{0-8 \mathrm{~h}}\right)$, estimated using the trapezoidal rule, the AUC extrapolated from time zero to infinity $\left(\mathrm{AUC}_{0-\text { inf }}\right.$ ), the highest observed plasma doxylamine concentration $\left(C_{\max }\right)$, the time to highest observed plasma doxylamine concentration $\left(t_{\max }\right)$, and the doxylamine elimination half-life $\left(t_{1 / 2}\right)$.

\subsection{Pharmacodynamics}

The pharmacodynamic evaluation included the Karolinska Sleepiness Scale (KSS) and psychomotor testing. The KSS is a 9-point assessment tool that includes descriptors of 1 for very alert, 3 for alert-normal level, 5 for neither alert nor sleepy, 7 for sleepy-but no effort to keep awake, and 9 for very sleepy, great effort to keep awake, fighting sleep [6]. Assessments were made before and at 10, 15, 20, 30, $45,60,75,90,120,150$, and 180 min after study drug dosing. Computer-administered psychomotor tests, including simple reaction time, choice reaction time, digit vigilance, and numeric working memory, were evaluated before and at 3, 4, 5, 6, 8, 12, and $24 \mathrm{~h}$ after study drug dosing using Cognitive Drug Research (CDR) system 1.3 software (United BioSource Corp., Blue Bell, PA, USA). The following pharmacodynamic parameters were collected: simple reaction time speed; digit vigilance speed, percentage targets detected, and number of false alarms; choice reaction time speed and percentage accuracy; numeric working memory sensitivity index and speed; power of attention (computed as the sum of simple reaction time, choice reaction time, and digit vigilance speed); and continuity of attention (computed as $0.45 \times$ digit variance targets detected $+0.50 \times$ choice reaction time accuracy - digit vigilance false alarms).

\subsection{Safety}

Adverse events (AEs) were monitored throughout the entire study and were evaluated by the investigator in terms of their intensity and relationship to study treatment. In addition, clinical laboratory testing, vital signs, 12-lead electrocardiograms, and physical examinations were performed at screening and at discharge from the study site.

\subsection{Statistics}

The sample size for this study was determined based on the number of subjects needed to derive pharmacokinetic profiles and was not sized for any statistical inferences. Subjects who received at least one dose of study medication were included in the safety analysis. Subjects who received study medication and did not have any protocol deviations that might affect plasma doxylamine concentrations were included in the pharmacokinetic analysis. Pharmacokinetic and pharmacodynamic parameters were evaluated for each study treatment using descriptive statistics. AEs were coded using the Medical Dictionary for Regulatory Activities. AEs and other safety parameters were summarized descriptively.

\section{Results}

\subsection{Subjects}

Eighty-nine subjects were screened for eligibility; of these, 24 subjects met eligibility criteria, were randomized to treatment sequence, and completed all four treatment periods. All 24 subjects were included in the pharmacokinetic and safety populations. The study population had a mean age of 35.9 years; $71 \%$ were male, and $96 \%$ were White (Table 1).

\subsection{Pharmacokinetics}

The mean plasma doxylamine-time curves by study treatment are shown in Fig. 1. Six of the 24 subjects (25\%) achieved the target plasma doxylamine concentration of $50 \mathrm{ng} / \mathrm{mL}$ following administration of the $12.7-\mathrm{mg}$ intranasal dose. In contrast, none of the subjects achieved this 
Table 1 Demographics of study subjects

\begin{tabular}{ll}
\hline Characteristic & Subjects $(n=24)$ \\
\hline Age (years) & \\
Mean (SD) & $35.9(8.00)$ \\
Median (range) & $33.5(25-58)$ \\
Gender, $n(\%)$ & \\
Male & $17(70.8)$ \\
Female & $7(29.2)$ \\
Race, $n(\%)$ & \\
White & $23(95.8)$ \\
American Indian or Alaska native & $1(4.2)$ \\
Height, mean (SD) (cm) & $168.0(9.5)$ \\
Weight, mean (SD) (kg) & $78.0(8.38)$ \\
\hline
\end{tabular}

$S D$ standard deviation

target with the two lower doxylamine intranasal doses, whereas all subjects reached the target with the 25-mg oral dose. Maximum plasma doxylamine concentrations were achieved approximately $2 \mathrm{~h}$ after intranasal or oral dosing (Table 2). The $C_{\max }$ and $\mathrm{AUC}_{0-8 \mathrm{~h}}$ increased with intranasal doxylamine dose in a slightly less than dose-proportional manner; at the 3.2-, 6.3-, and 12.7-mg doses, mean $C_{\max }$ values were $12.6,18.7$, and $32.9 \mathrm{ng} / \mathrm{mL}$, respectively, and mean $\mathrm{AUC}_{0-8 \mathrm{~h}}$ values were 74.8, 111.2, and $192.6 \mathrm{ng} \cdot \mathrm{h} /$
$\mathrm{mL}$, respectively. At the highest intranasal dose evaluated, the $C_{\max }$ and $\mathrm{AUC}_{0-8 \mathrm{~h}}$ were approximately $25 \%$ of the values achieved with the $25-\mathrm{mg}$ oral dose. The mean $\mathrm{AUC}_{0-\text { inf }}$ following the 12.7-mg intranasal dose was onethird of that following the 25-mg oral dose (632 and $1865 \mathrm{ng} \cdot \mathrm{h} / \mathrm{mL}$, respectively). Mean $t_{1 / 2}$ did not differ across study treatments, ranging from 10.2 to $11.4 \mathrm{~h}$ for the intranasal doses and $10.3 \mathrm{~h}$ for the oral dose. Notably, the coefficient of variation for most of the pharmacokinetic parameters was much higher with the intranasal doses than with the oral dose.

\subsection{Pharmacodynamics}

Mean baseline scores on the KSS ranged from 2.7 to 3.3 across the four treatment periods. Mean KSS scores increased following administration of study treatment, generally reaching a maximum at $90 \mathrm{~min}$ post-dose (Fig. 2). Changes from baseline in KSS were consistent with the doxylamine pharmacokinetics. The magnitude of the effect increased with intranasal dose (change from baseline: $1.5,1.8$, and 2.6 for the 3.2-, 6.3-, and 12.7-mg doses, respectively) and was greatest with the $25-\mathrm{mg}$ oral dose (change from baseline: 3.2 ). The pharmacokineticpharmacodynamic relationship was evaluated for each treatment. The correlation between plasma doxylamine concentrations versus the change from baseline in KSS

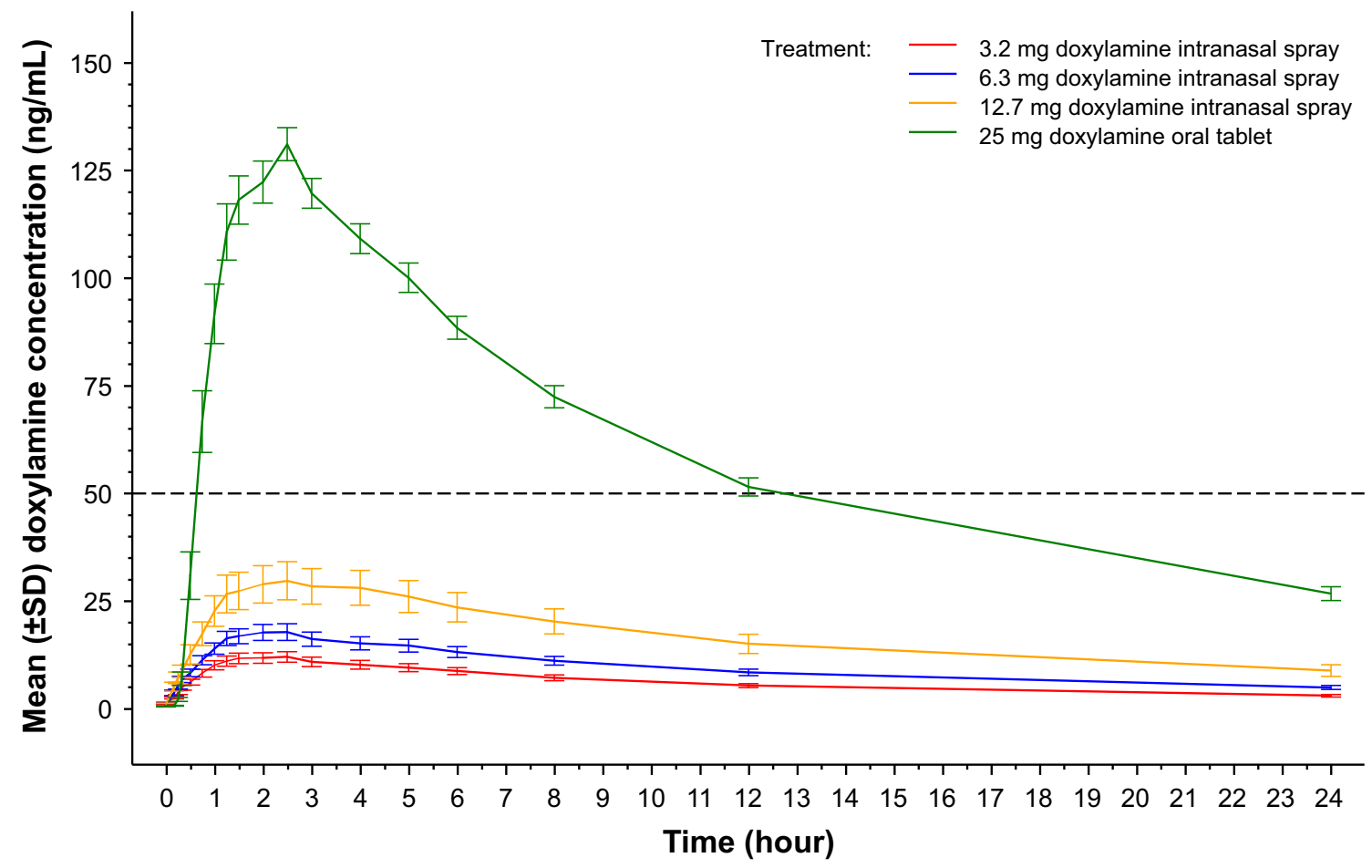

Fig. 1 Mean plasma doxylamine concentration-time curves by treatment group. The horizontal line indicates the target plasma doxylamine concentration of $50 \mathrm{ng} / \mathrm{mL}$. SD standard deviation 
Table 2 Pharmacokinetic parameters, pharmacokinetic analysis population

\begin{tabular}{|c|c|c|c|c|}
\hline \multirow[t]{3}{*}{ Parameter } & \multicolumn{4}{|l|}{ Doxylamine dose } \\
\hline & \multicolumn{3}{|l|}{ Intranasal spray } & \multirow[t]{2}{*}{ Oral tablet $25 \mathrm{mg}(n=24)$} \\
\hline & $3.2 \mathrm{mg}(n=24)$ & $6.3 \mathrm{mg}(n=24)$ & $12.7 \mathrm{mg}(n=24)$ & \\
\hline \multicolumn{5}{|c|}{$\mathrm{AUC}_{0-8 \mathrm{~h}}(\mathrm{ng} \cdot \mathrm{h} / \mathrm{mL})$} \\
\hline Mean (SD) & $74.8(35.13)$ & $111.2(52.37)$ & $192.6(133.15)$ & $750.0(95.52)$ \\
\hline Median (range) & $81.1(19.1-128.1)$ & $117.7(40.9-232.3)$ & $161.8(36.6-476.4)$ & $751.8(583.0-898.5)$ \\
\hline $\mathrm{CV}(\%)$ & 46.9 & 47.1 & 69.1 & 12.7 \\
\hline \multicolumn{5}{|l|}{$C_{\max }(\mathrm{ng} / \mathrm{mL})$} \\
\hline Mean (SD) & $12.6(6.20)$ & $18.7(9.53)$ & $32.9(22.96)$ & $137.9(17.39)$ \\
\hline Median (range) & $12.9(2.7-21.9)$ & $18.9(6.0-38.4)$ & $27.1(5.1-82.0)$ & $142.0(96.5-164.0)$ \\
\hline $\mathrm{CV}(\%)$ & 49.3 & 50.9 & 69.7 & 12.6 \\
\hline \multicolumn{5}{|l|}{$t_{\max }(\mathrm{h})$} \\
\hline Mean (SD) & $2.0(0.89)$ & $2.0(0.91)$ & $2.3(1.08)$ & $2.1(0.50)$ \\
\hline Median (range) & $2.0(0.8-5.0)$ & $2.0(0.3-5.0)$ & $2.5(0.3-5.0)$ & $2.0(1.3-3.0)$ \\
\hline $\mathrm{CV}(\%)$ & 44.8 & 45.1 & 47.5 & 23.8 \\
\hline$t_{1 / 2}(\mathrm{~h})$ & $(n=15)$ & $(n=14)$ & $(n=11)$ & $(n=23)$ \\
\hline Mean (SD) & $10.2(1.49)$ & $11.4(1.31)$ & $10.3(0.85)$ & $10.3(1.59)$ \\
\hline Median (range) & $10.2(8.0-12.7)$ & $11.8(8.5-12.8)$ & $10.1(9.4-12.2)$ & $10.4(7.3-12.8)$ \\
\hline CV (\%) & 14.5 & 11.6 & 8.3 & 15.5 \\
\hline
\end{tabular}

$A U C_{0-8 h}$ area under the plasma doxylamine concentration-time curve between time zero and $8 \mathrm{~h}, C_{\max }$ maximum plasma doxylamine concentration, $C V$ coefficient of variation, $S D$ standard deviation, $t_{1 / 2}$ doxylamine elimination half-life, $t_{\max }$ time to maximum plasma doxylamine concentration

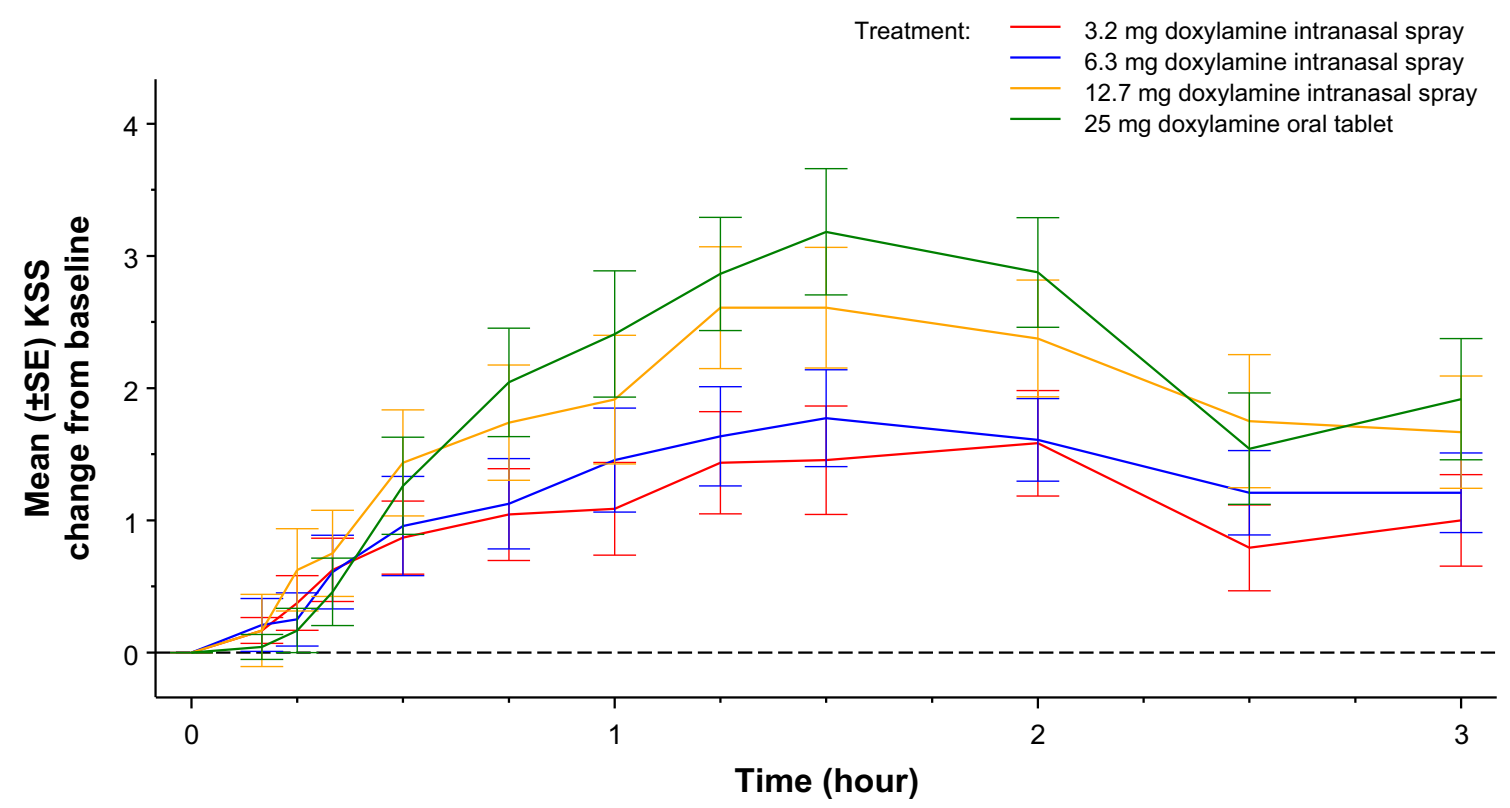

Fig. 2 Mean changes from baseline $( \pm \mathrm{SE})$ in KSS scores over time by treatment group. KSS Karolinska Sleepiness Scale, $S E$ standard error

score was best demonstrated for the 25-mg oral dose. However, a drug concentration-effect relationship was also evident for the intranasal doses, particularly for $12.7 \mathrm{mg}$, where changes in KSS were associated with smaller increases in plasma doxylamine concentration than were observed with the oral dose (Fig. 3a, b).

Changes from baseline in psychomotor parameters were generally variable over time, did not show a relationship to intranasal dose, and did not distinguish between intranasal 

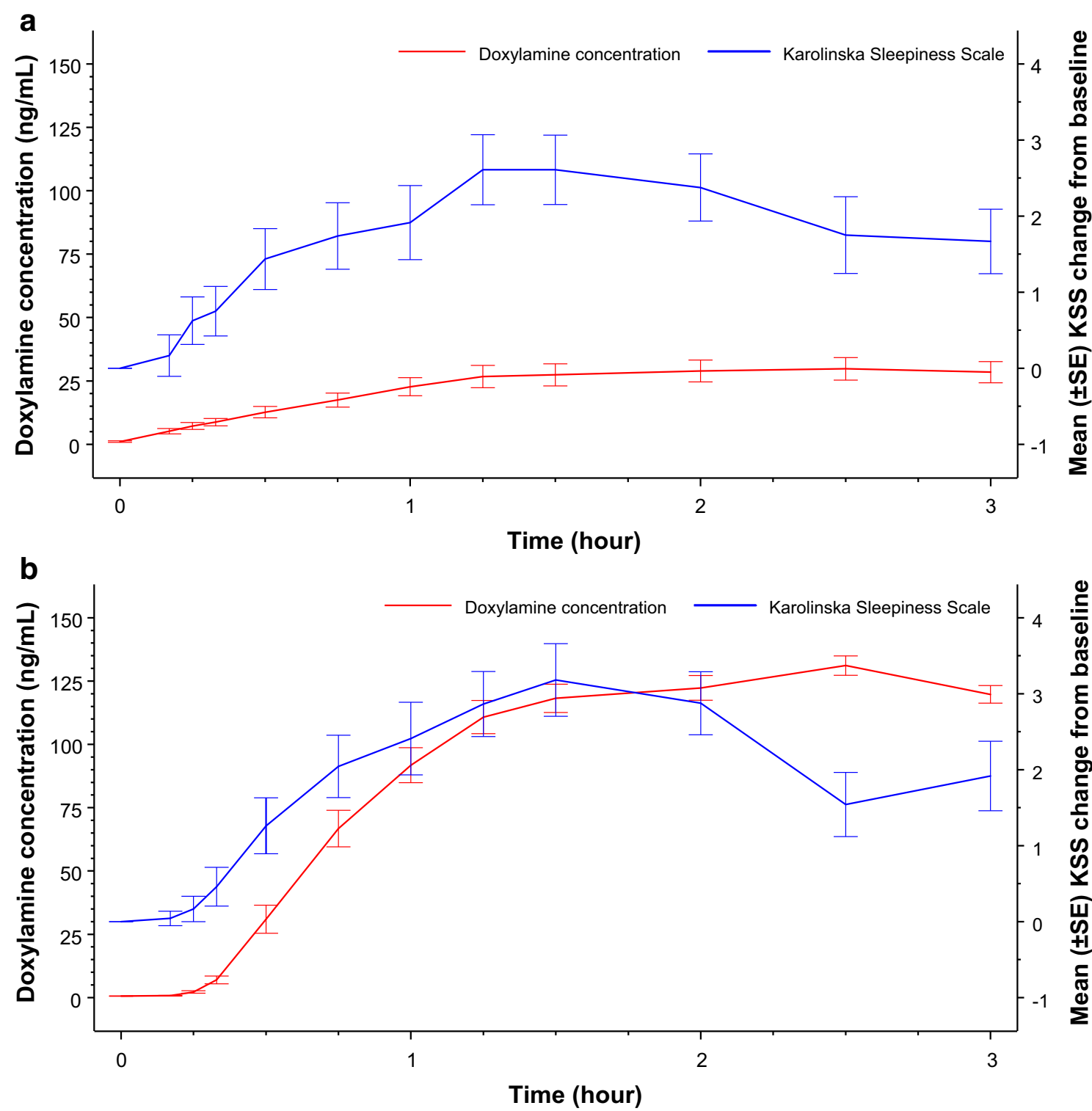

Fig. 3 Comparison of changes from baseline in KSS scores with plasma doxylamine concentration over time following administration of the 12.7-mg doxylamine nasal spray (a) and 25-mg doxylamine oral tablet (b). KSS Karolinska Sleepiness Scale, SE standard error

versus oral dosing. The mean changes from baseline in simple reaction time and digit vigilance speed are shown in Fig. 4a, b.

\subsection{Safety}

A total of 14 AEs were observed in 10 subjects who received intranasal doxylamine; all were rated as mild in intensity and were transient or intermittent. The most common AEs were nasal congestion $(n=4)$, nasal dryness $(n=2)$, and frontal headache $(n=2)$, which were all observed following administration of an intranasal dose. No serious AEs were observed. No significant changes in clinical laboratory values or other safety parameters were observed during the study. No AEs were reported for subjects who received oral doxylamine.

\section{Discussion}

This study was conducted to select an appropriate dose of the doxylamine succinate nasal spray for subsequent clinical evaluation compared with the existing 25-mg OTC tablet. The results of the pharmacokinetic analysis demonstrated that intranasal administration of doxylamine succinate at doses of 3.2 and $6.3 \mathrm{mg}$ did not achieve the target plasma concentration of $50 \mathrm{ng} / \mathrm{mL}$, whereas the 12.7-mg intranasal dose achieved the target concentration 


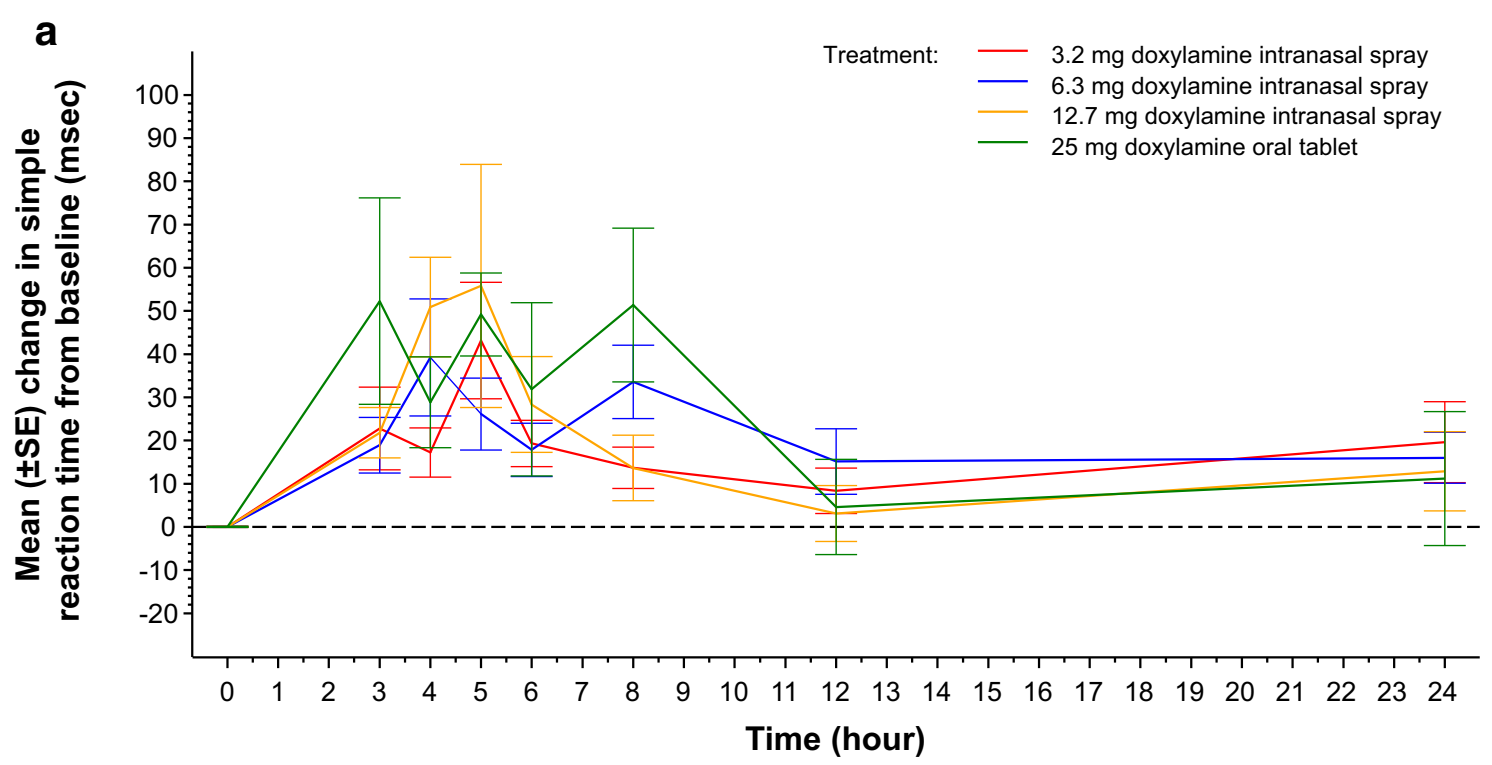

b

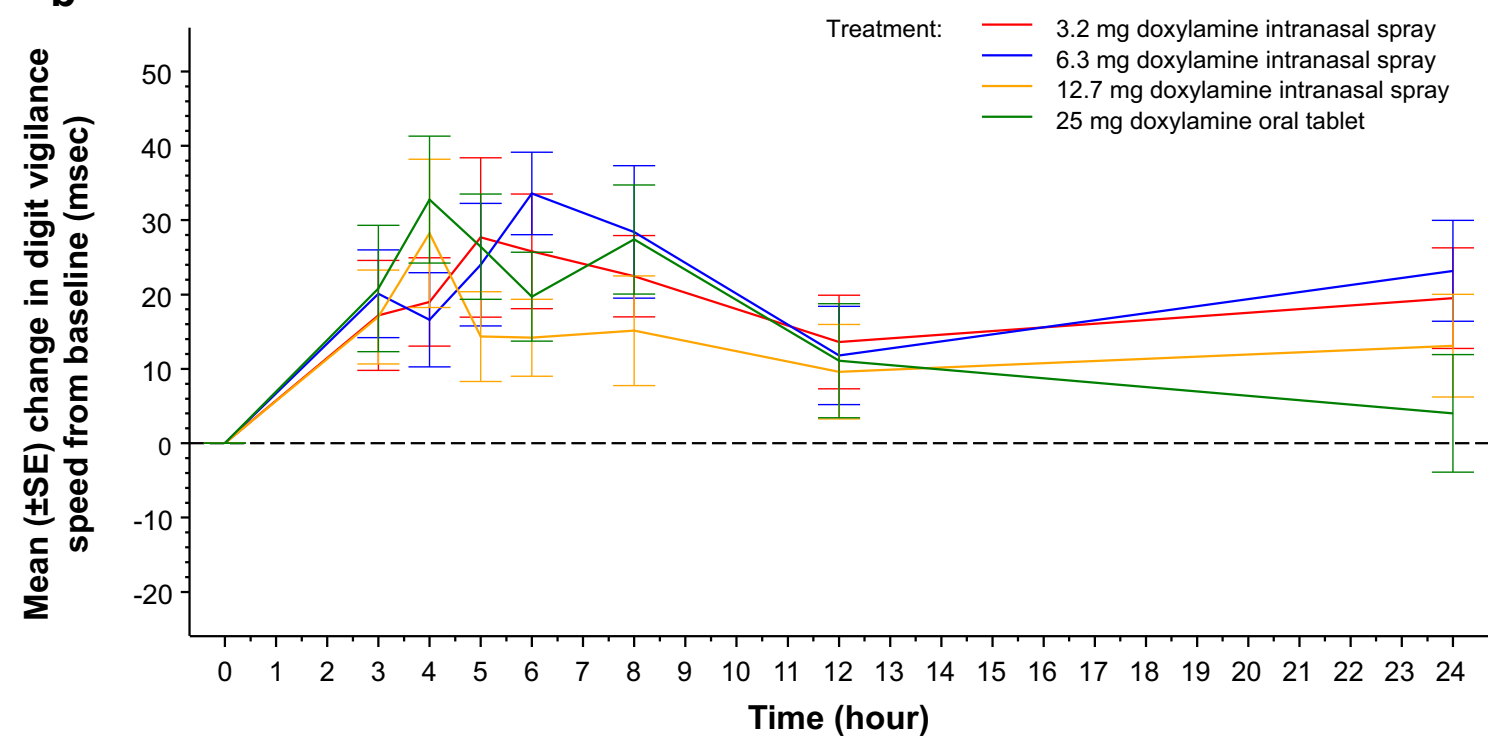

Fig. 4 Mean changes from baseline $( \pm \mathrm{SE})$ in simple reaction time (a) and digit vigilance speed (b) over time by treatment group. $S E$ standard error

in only $25 \%$ of subjects. Moreover, the $\mathrm{AUC}_{0-8 \mathrm{~h}}$ values following intranasal administration of doxylamine succinate were lower than that obtained following oral dosing, indicating that intranasal bioavailability at the three dose levels tested was lower than that for the existing 25-mg OTC tablet. These findings differ from a previous study conducted in rats, which showed that intranasal administration resulted in more rapid absorption and higher bioavailability compared with oral administration [5].

Pharmacokinetic data for orally administered doxylamine succinate are limited. A study in 16 healthy male volunteers given a single 25-mg tablet of doxylamine succinate with $240 \mathrm{~mL}$ of water found a mean $C_{\max }$ of
$99 \mathrm{ng} / \mathrm{mL}$, with individual values ranging from 60 to $138 \mathrm{ng} / \mathrm{mL}$ [7]. The mean $C_{\max }$ of $137.9 \mathrm{ng} / \mathrm{mL}$ (range $96.5-164.0 \mathrm{ng} / \mathrm{mL}$ ) reported herein with the $25-\mathrm{mg}$ oral OTC tablet was somewhat higher, possibly reflecting differences in oral tablet formulation and study population. However, the $t_{\max }$ of $2.4 \mathrm{~h}$ and $t_{1 / 2}$ of $10.1 \mathrm{~h}$ reported in healthy male volunteers in the previous study [7] are similar to the levels found in our study (mean $t_{\max }$ of $2.1 \mathrm{~h}$ and $t_{1 / 2}$ of $10.3 \mathrm{~h}$ ). No other study of the pharmacokinetics of doxylamine succinate oral tablets in adults was found in a MEDLINE search using the terms 'doxylamine' and 'pharmacokinetics'. 
Changes from baseline in KSS scores were generally consistent with the pharmacokinetics of doxylamine succinate. The effect on KSS increased with the intranasal dose, and the magnitude of the effect was greatest with the oral dose. However, the psychomotor parameters generally varied over time, did not show a relationship to the intranasal dose, and did not distinguish between the intranasal spray and OTC tablet. As a result, a proper dose-effect relationship could not be established for the doxylamine succinate nasal spray formulation.

It is unclear whether the lower bioavailability of intranasal doxylamine succinate is due to the route of administration, the formulation, or some other factor or combination of factors. One could speculate that the inclusion of a permeation enhancer could maximize the systemic bioavailability of intranasal doxylamine succinate. These enhancers, such as the commercially available dodecyl maltoside (Intravail ${ }^{\circledR}$; Aegis Therapeutics, LLC, San Diego, CA, USA), are designed to improve the absorption of pharmacologically active drug by enhancing membrane permeability rather than increasing solubility, as has been demonstrated independently for both intranasal sumatriptan and hydromorphone [8-10].

\section{Conclusion}

In summary, although the pharmacokinetics of the doxylamine succinate nasal spray appeared dose-related, none of the three dose levels produced a mean $C_{\max }$ above the target level of $50 \mathrm{ng} / \mathrm{mL}$ or a $t_{\max }$ shorter than that of the existing OTC tablet. Therefore, the nasal spray at the doses tested does not increase the absorption or systemic bioavailability of doxylamine succinate compared with the 25-mg oral tablet. Future studies with higher dose levels of doxylamine succinate nasal spray should be considered.

\begin{abstract}
Acknowledgments This study was sponsored by GlaxoSmithKline Consumer Healthcare. Medical writing assistance was provided by Barry Weichman, $\mathrm{PhD}$, and Kristin Larsen, $\mathrm{PhD}$, of Peloton Advantage and was funded by GlaxoSmithKline Consumer Healthcare. GlaxoSmithKline Consumer Healthcare provided a full review of the article.
\end{abstract}

\section{Compliance with Ethical Standards}

Conflict of interest Mark Allison is an employee of Celerion. Cecilia Hale is an employee of Hurley Consulting Associates and was an employee of GlaxoSmithKline at the time of study conduct.
Research involving human participants The study was conducted in accordance with ethical principles originating in the Declaration of Helsinki and in compliance with the International Council for Harmonisation Guideline for Good Clinical Practice and all applicable regulations. The study protocol was approved by Celerion Institutional Review Board before subjects were enrolled.

Informed consent Informed consent was obtained from all individual participants included in the study.

Open Access This article is distributed under the terms of the Creative Commons Attribution-NonCommercial 4.0 International License (http://creativecommons.org/licenses/by-nc/4.0/), which permits any noncommercial use, distribution, and reproduction in any medium, provided you give appropriate credit to the original author(s) and the source, provide a link to the Creative Commons license, and indicate if changes were made.

\section{References}

1. Sjoqvist F, Lasagna L. The hypnotic efficacy of doxylamine. Clin Pharmacol Ther. 1967;8:48-54.

2. Food and Drug Administration. Over-the-counter nighttime sleep-aid and stimulant products. Fed Regist. 1978;43:25544-602.

3. Culpepper L, Wingertzahn MA. Over-the-counter agents for the treatment of occasional disturbed sleep or transient insomnia: a systematic review of efficacy and safety. Prim Care Companion CNS Disord. 2015;17(6).

4. Vande Griend JP, Anderson SL. Histamine-1 receptor antagonism for treatment of insomnia. $\mathrm{J}$ Am Pharm Assoc. 2012;52:e210-9.

5. Pelser A, Muller DG, du Plessis J, du Preez JL, Goosen C. Comparative pharmacokinetics of single doses of doxylamine succinate following intranasal, oral and intravenous administration in rats. Biopharm Drug Dispos. 2002;23:239-44.

6. Akerstedt T, Anund A, Axelsson J, Kecklund G. Subjective sleepiness is a sensitive indicator of insufficient sleep and impaired waking function. J Sleep Res. 2014;23:242-54.

7. Friedman H, Greenblatt DJ. The pharmacokinetics of doxylamine: use of automated gas chromatography with nitrogenphosphorus detection. J Clin Pharmacol. 1985;25:448-51.

8. Aungst BJ. Absorption enhancers: applications and advances. AAPS J. 2012;14:10-8.

9. Cunningham G, Maggio ET. Non-invasive nasal hydromorphone with high bioavailability for rapid onset and non-dissociative acute pain control: a feasibility study. J Bioequiv Availab. 2018;10:14-7.

10. Munjal S, Gautam A, Offman E, Brand-Schieber E, Allenby K, Fisher DM. A randomized trial comparing the pharmacokinetics, safety, and tolerability of DFN-02, an intranasal sumatriptan spray containing a permeation enhancer, with intranasal and subcutaneous sumatriptan in healthy adults. Headache. 2016;56:1455-65. 